\title{
The $\delta$ Scuti star 44 Tau: Observations and analysis
}

\section{Victoria Antoci and Michel Breger}

Institut for Astronomy, Vienna University, Türkenschanzstrasse 17, A-1180, Vienna, Austria e-mail: antoci@astro.univie.ac.at

Abstract. Between 2000 and 2003 more than 600 hours of photometric data of the $\delta$ Scuti star 44 Tauri (HD 26322) were collected in Strömgren $v$ and $y$ as well as in Johnson $V$. Observations were carried out at Washington Camp (Arizona), the Sierra Nevada Observatory (Spain) and the Ege University Observatory (Turkey). After analyzing the data with Period98 (Sperl 1998) more than 30 frequencies were found to be significant. We present the data and the results of our observational campaigns.

Keywords. Methods: data analysis, techniques: photometric, stars: oscillations (including pulsations), stars: individual: (44 Tau)

\section{Introduction}

$\delta$ Scuti stars are pulsating variables of spectral types A to $\mathrm{F}$ of luminosity class III to V. They are situated in the lower part of the Classical Instability Strip on and above the hydrogen-burning Main Sequence, the adult stage of stellar evolution. Most of these stars pulsate simultaneously in radial and nonradial modes, which makes them excellent candidates for asteroseismic studies.

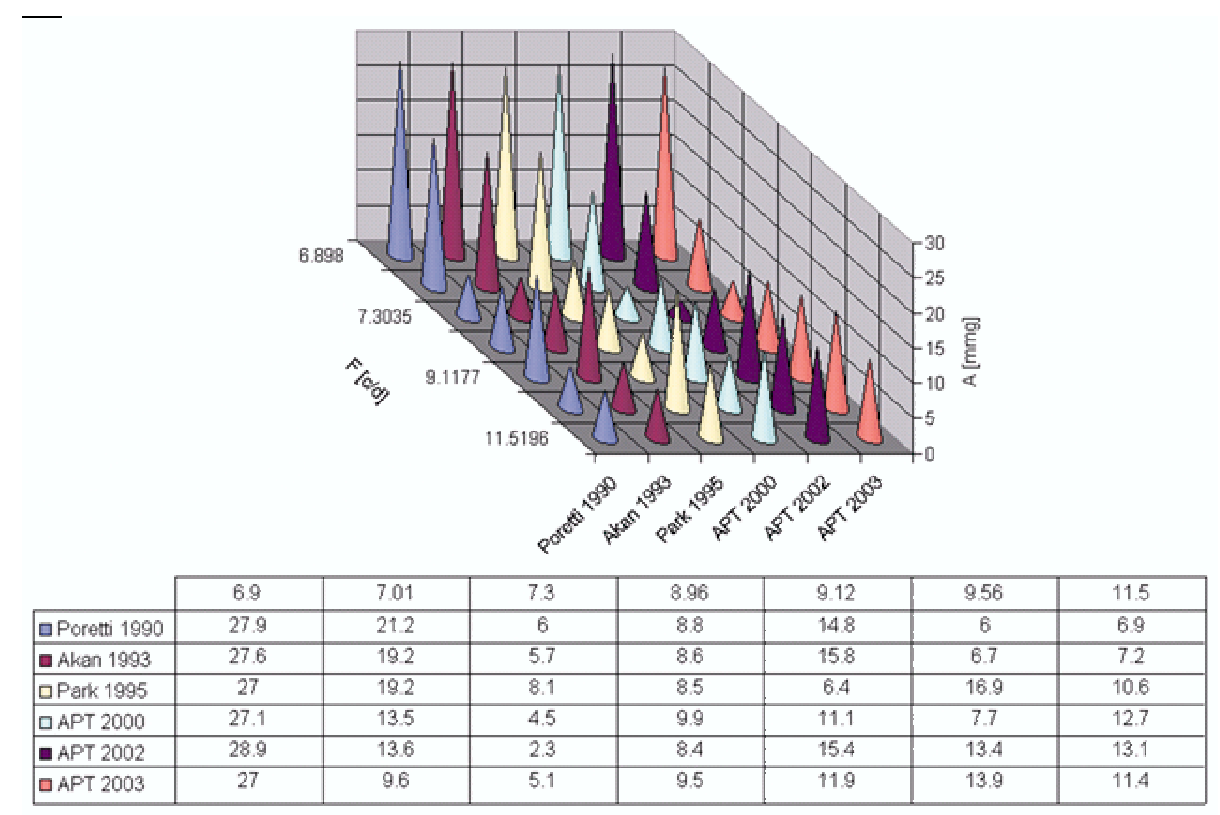

Figure 1. Amplitude variations between 1990 and 2003 in Strömgren $y$. 


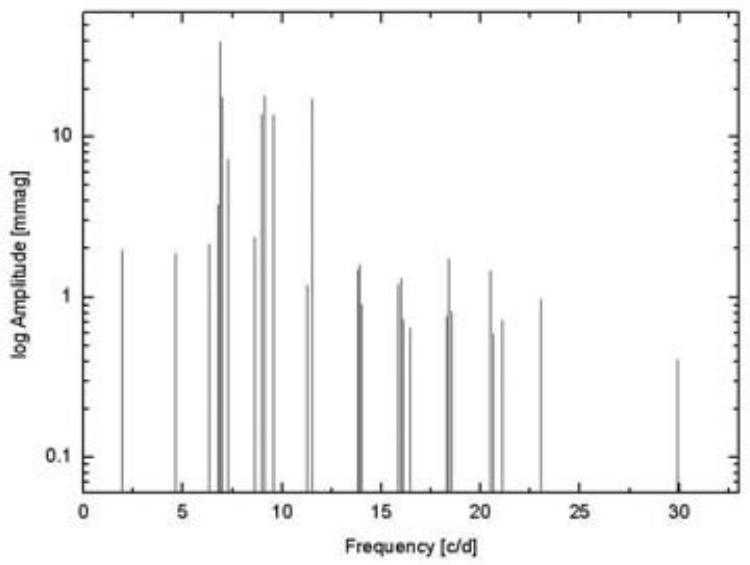

Figure 2. Frequency spectrum of 44 Tau in Strömgren $v$.

With a very small value of $v \sin i=6.8 \pm 1.6 \mathrm{~km} \mathrm{~s}^{-1}$ (Solano \& Fernley 1997), 44 Tau (spectral type F2 IV) is one of the most promising candidates for asteroseismology. The star rotates either very slowly or it is seen almost pole-on. In the case of slow rotation,

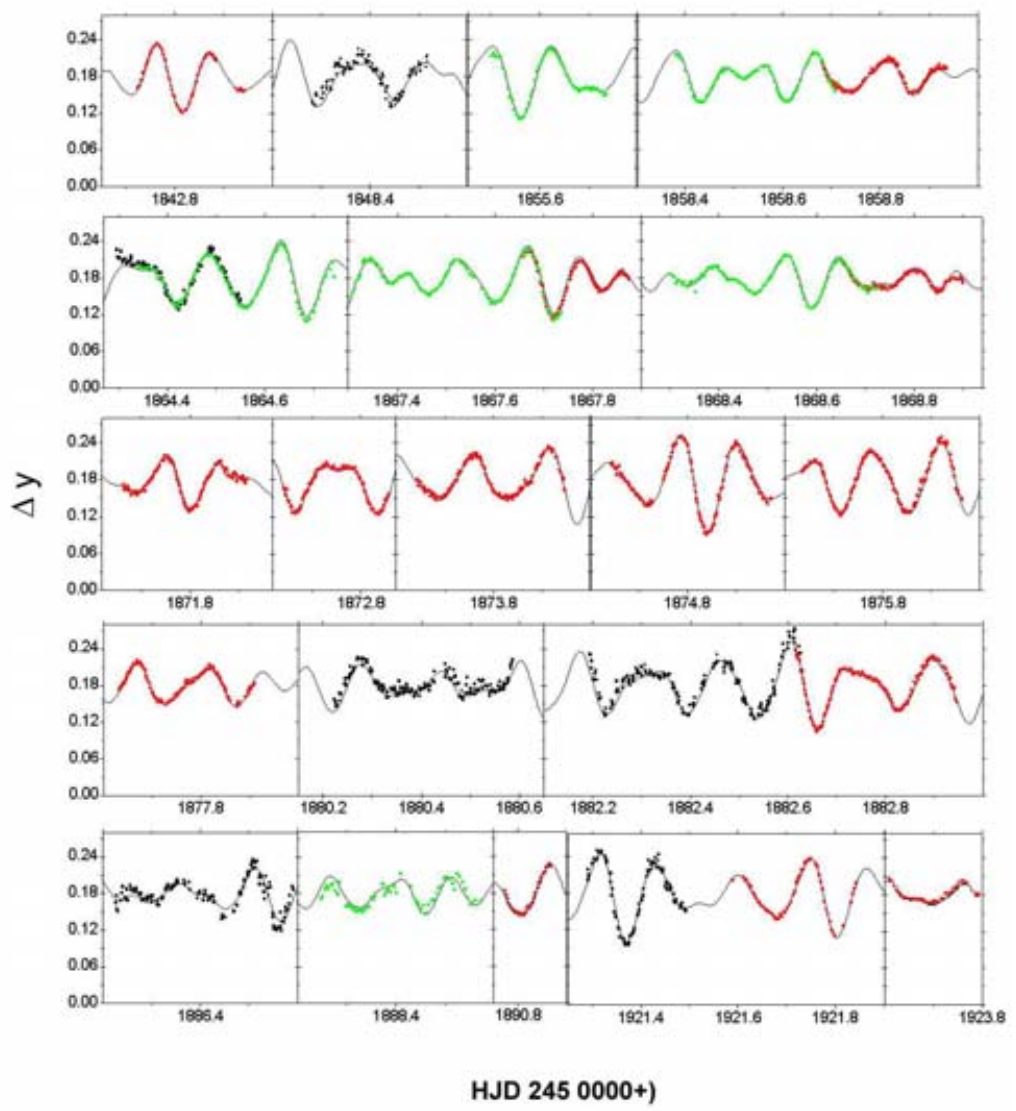

Figure 3. Multisite photometry of 44 Tau obtained during the DSN campaign 2000/2001. The fit is shown as a solid curve. Red dots: APT, green dots: Sierra Nevada Observatory, black dots: Ege University Observatory. 
Table 1. Frequencies of 44 Tauri in Strömgren $v$ and $y$.

\begin{tabular}{lrcc}
\hline & $F(\mathrm{c} / \mathrm{d})$ & $A_{v}(\mathrm{mmag})$ & $A_{y}(\mathrm{mmag})$ \\
\hline$f 1$ & 6.8980 & 39.06 & 26.75 \\
$f 2$ & 7.0060 & 17.74 & 12.43 \\
$f 3$ & 9.1175 & 17.91 & 12.23 \\
$f 4$ & 11.5196 & 17.25 & 12.20 \\
$f 5$ & 8.9606 & 14.00 & 9.71 \\
$f 6$ & 9.5613 & 13.72 & 9.09 \\
$f 7$ & 7.3034 & 7.20 & 4.72 \\
$f 8$ & 6.7952 & 3.76 & 2.95 \\
$f 9$ & 9.5803 & 2.44 & 1.63 \\
$f 10$ & 6.3390 & 2.14 & 1.75 \\
$f 11$ & 8.6421 & 2.36 & 2.04 \\
$f 12$ & 11.2920 & 1.17 & .77 \\
$f 4-f 6$ & 1.9583 & 1.94 & $S / N<3.5$ \\
$f 1+f 4$ & 18.4176 & 1.71 & 1.14 \\
$2 f 1$ & 13.7961 & 1.47 & 1.11 \\
$f 4+f 5$ & 20.4802 & 1.44 & 1.17 \\
$f 1+f 2$ & 13.9040 & 1.57 & .80 \\
$f 1+f 5$ & 15.8586 & 1.18 & .84 \\
$f 1+f 3$ & 16.0155 & 1.30 & .99 \\
$2 f 4$ & 23.0393 & .98 & .73 \\
$2 f 2$ & 14.0120 & .91 & .69 \\
$f 2+f 4$ & 18.5256 & .81 & .63 \\
$f 2+f 3$ & 16.1235 & .73 & $S / N<3.5$ \\
$f 4+f 6$ & 21.0809 & .72 & $S / N<3.5$ \\
$f 4+f 8$ & 18.3149 & .76 & .60 \\
$f 1+f 6$ & 16.4593 & .64 & $S / N<3.5$ \\
$f 3+f 4$ & 20.6371 & .59 & $S / N<3.5$ \\
$2 f 4+f 1$ & 29.9373 & .41 & $S / N<3.5$ \\
$f 4-f 1$ & 4.6216 & $S / N<3.5$ & 1.25 \\
\hline & & &
\end{tabular}

the overlap of rotationally split modes of different orders is prevented, so that mode identification is simplified. Furthermore the small rotational velocity gives rise to easily recognizable equidistant multiplets.

\section{Photometry}

To acquire the photometric data, the three-star technique (Breger 1993) was adopted, in which measurements of the variable star are alternated with those of the comparison stars HD 25867 and HD 25678, for which no variability has been detected.

\section{Frequency detection}

The combined photometric data were searched for periodic signals using Period98 (a package based on Fourier and multiple-least-squares techniques). Twenty-nine frequencies were found to be significant adopting the amplitude signal to noise (Breger 1993) criterion of 4.0, and for linear combination frequencies a $S / N$ of 3.5. Furthermore, amplitude variations could be detected (Fig. 1). Table 1 summarizes the results. 


\section{Mode identification}

The identification of $f 1$ as the fundamental radial mode and of $f 2$ as its first overtone is suggested by the agreement of the period ratio model 3.1 by Stellingwerf (1979). These results also correspond to those of Kirbiyk (2003), Civelek (2001) and Poretti (1992).

\section{Future work}

Theoretical line-profile calculations have shown that, although the star is slowly rotating, spectroscopic mode identification is possible. As for FG Vir, due to the small value of the projected rotational velocity, photometry and spectroscopy are expected to reveal the same frequencies, which helps to disentangle the different modes. The goal of the next observing campaign (starting next autumn) is the collection of sufficient photometric and spectroscopic data which will enable us to identify additional oscillation modes and to calculate theoretical models of 44 Tau.

\section{Acknowledgements}

This work was supported by the FWF, P14546-PHY project

\section{References}

Akan, M.C. 1993, A\& A 278, 150

Breger, M. 1993, in: C.J. Buttler \& I. Elliott (eds), Stellar Photometry - Current Techniques and Future Developments, (Cambridge University Press), p. 106

Civelek, R., Kiziloglu, N., Kirbyik, H. 2001, AJ 122, 2042

Kirbyik, H., Civelek, R., Kiziloglu, N. 2003, ASS 288, 305

Park, N.K., Lee, S.W. 1995, AJ 109, 774

Poretti, E., Mantegazza, L., Riboni, E. 1992, A\&A 256, 113

Solano, E. \& Fernley, J. 1997, A\& AS 122, 131

Sperl, M. 1998, Comm. in Asteroseismology (Vienna) 111, 1

Stellingwerf, R.F. 1979, ApJ 227, 935 